\title{
Deciduoid Mesothelioma: Cytologic Presentation and Diagnostic Pitfalls
}

\author{
Cheng Cheng Huang, M.D. and Claire W. Michael, M.D.*
}

We report two cases of malignant deciduoid mesothelioma $(M D M)$, a very rare variant of malignant mesothelioma (MM). Case 1: An 18-year-old male with no history of asbestos exposure presented with worsening abdominal pain, anorexia, and vomiting after a motor vehicle accident. A CT scan showed small amount of ascites and abdominal mass. An exploratory laparotomy revealed multiple yellow tan, firm nodules on the peritoneum and omentum. He received palliative treatment and died 5 months after the diagnosis. Case 2: A 64-year-old female with history of asbestos exposure initially presented with abdominal distension. CT scan showed abdominal mass with a large amount of ascites. A diagnostic laparoscopy revealed multiple peritoneal nodules. She underwent several regimens of chemotherapy over a period of 69 months and is still alive to date. In both cases, features of mesothelial origin were subtle and the smears showed predominantly single cells with marked nuclear atypia. The second case also contained few two-dimensional loose cell clusters with scalloped or hobnail borders. The clusters often exhibited a pseudoacinar structure surrounding a globular extracellular material. Groups of three to four cells often formed doublets and triplets with cell-to-cell windows.

Our results show that MDM may not present with the traditional cytological features described in MM and can manifest with more nuclear pleomorphism resulting in erroneous diagnosis. Recognition of the subtle mesothelial features along with the appropriate ancillary tests is essential for accurate diagnosis. Diagn. Cytopathol. 2013;41:629-635. @ 2012 Wiley Periodicals, Inc.

Key Words: cytology; malignant deciduoid mesothelioma; peritoneal effusion

Malignant deciduoid mesothelioma (MDM) is a very rare variant of mesothelioma. A total of $44 \mathrm{MDM}$ cases have

Department of Pathology, University of Michigan, Ann Arbor, Michigan

*Correspondence to: Claire W. Michael, M.D., Professor, Department of Pathology, Case Western Reserve University/University Hospitals Case Medical Center, 11100 Euclid Ave Rm 212B, Cleveland, OH 44106, USA. E-mail: claire.michael@uhhospitals.org

Received 29 February 2012; Accepted 24 June 2012

DOI 10.1002/dc. 22902

Published online 25 September 2012 in Wiley Online Library

(wileyonlinelibrary.com). been documented in the English literature since this entity was first described in $1985 .^{1,2}$ In a study of 320 malignant mesothelioma (MM) cases, only $4(1.25 \%)$ were subclassified as MDM. ${ }^{3}$ Most of these reports focused on the histological features of MDM. ${ }^{2-8}$ To date, the cytomorphology of MDM has only been described in three single case reports, in which specimens from fine needle aspiration, ${ }^{1}$ peritoneal fluid, ${ }^{9}$ and pericardial fluid ${ }^{10}$ were studied. In the present study, we report the cytological features in two histologically confirmed peritoneal MDM cases presenting with ascites.

\section{Materials and Methods}

The standard laboratory methods were used in preparation of the slides for serous fluid, with the preparation of two air-dried and Diff-Quik-stained smears, two fixed and Papanicolaou-stained smears and a cell block. All surgical biopsies and cell block cell pellets were fixed in $10 \%$ buffered formalin and embedded in paraffin. The paraffin embedded blocks were cut into 4 - $\mu$ m-thick sections and stained with Hematoxylin and Eosin (H\&E). The immunohistochemical staining was performed on sections of the cell blocks according to standard laboratory protocols.

\section{Clinical Presentations and Pathological Findings}

Case 1. An 18-year-old white male who suffered from a side impact motor vehicle accident on December 15, 1997 was sent home without a specified injury. Since that time he has had vague abdominal pain, anorexia, and vomiting. He presented to our hospital on January 13, 1998 with increased abdominal pain and anorexia. A CT scan of the abdomen and pelvis showed small amount of ascites and multiple nodules on the surface of the peritoneum and omentum. He was taken to the operating room with a presumptive acute surgical abdomen on January 14, 1998. An exploratory laparotomy revealed multiple yellow tan, firm nodules on the peritoneum and omentum. 


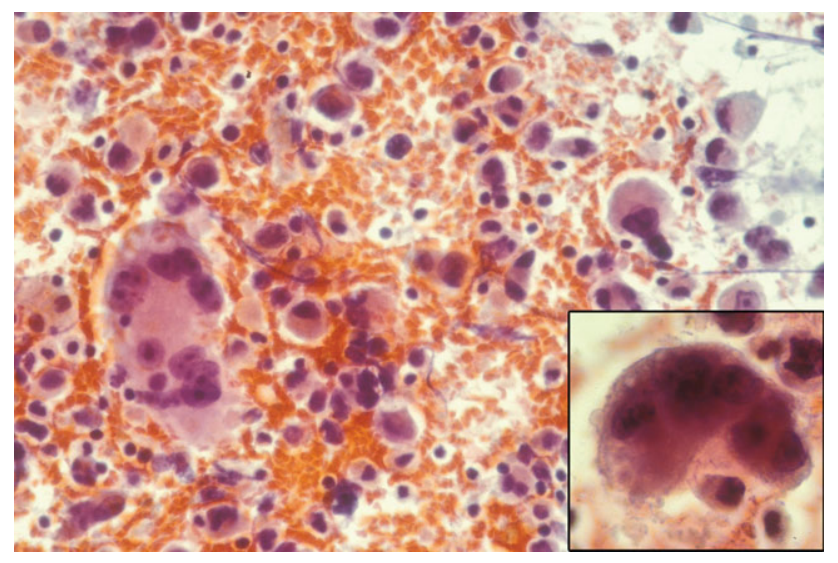

Fig. 1. Case 1: The single cells are round, oval or polygonal shaped with wide variation in size and striking nuclear atypia. A multinucleated cell shows nuclei situated at opposite poles of the cell. Cell exhibits submembranous glycogen vacuoles and brush-like border (insert). Diff-Quik stain, $\times 600$. [Color figure can be viewed in the online issue, which is available at wileyonlinelibrary.com.]

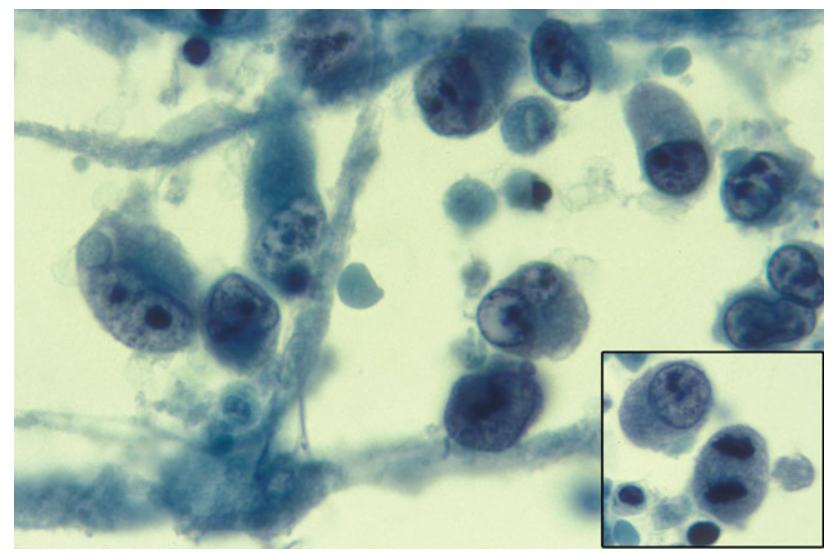

Fig. 2. Case 1: The nuclei are round, oval or indented with irregularly clumped chromatin, and prominent macronucleoli. Mitotic figures are frequently seen (insert). Papanicolaou stain, $\times 600$. [Color figure can be viewed in the online issue, which is available at wileyonlinelibrary.com.]

An omental tissue biopsy was performed and initially diagnosed as exuberant reactive changes featuring a prominent mesothelial component. Recurrent ascites was aspirated on January 19, 1998, which was clearly malignant in nature triggering a second review of the biopsy and the ultimate diagnosis of MDM. Consequently, the patient developed worsening abdominal pain and ascites. He was referred to hospice care where he was managed by palliative treatment, and announced dead on May 31, 1998. He had no history of asbestos exposure. However, his uncle and grandmother both had history of mesothelioma.

A total $8 \mathrm{ml}$ of bloody ascites was obtained by peritoneocentesis for cytology. The bloody smears (Fig. 1) were moderate in cellularity with predominantly a single cell pattern and rare scattered small clusters. Overall the cells were widely variable in size and shape with striking nu-

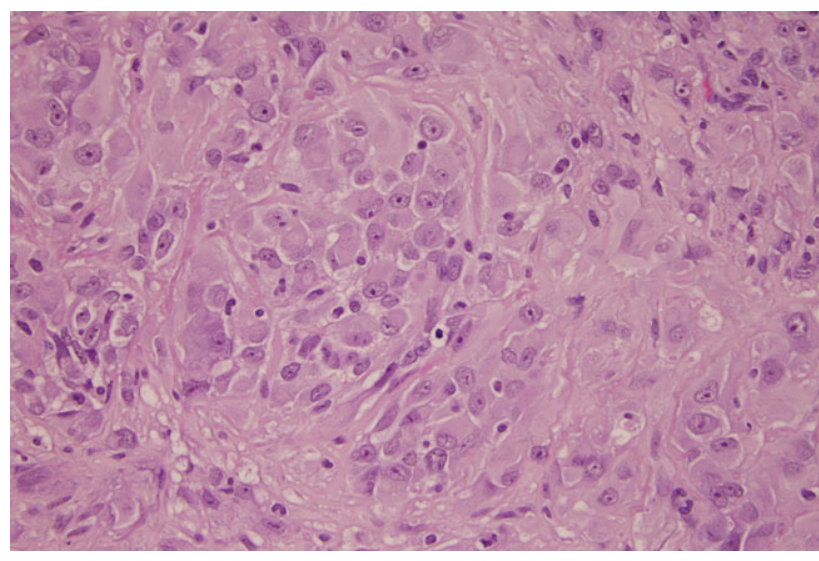

Fig. 3. Case 1: The polygonal shaped cells are large with voluminous, bright eosinophilic to glassy cytoplasm. The nuclei are round to oval, with vesicular chromatin and eosinophilic macronucleoli. H\&E stain, $\times 400$. [Color figure can be viewed in the online issue, which is available at wileyonlinelibrary.com.]

clear atypia. The cells exhibited several shapes including: round, oval, and polygonal. While mostly singly scattered, occasional doublets and triplets were seen with vague articulation between the cells (windows). However, cellular clasping was not detected. Cell-in-cell was occasionally seen. Many tumor cells were large with voluminous cytoplasm resulting in low $N / C$ ratio in most cells. The cytoplasm was dense and eosinophilic with no discrete variation in tone or endo-ectoplasmic demarcation. Submembranous glycogen vacuoles and brush-like borders were identified at high magnification in occasional cells (Fig. 1, insert). Binucleated or multinucleated cells were commonly present and frequently the nuclei were situated at opposite poles of the cell (Fig. 1). The nuclei were round, oval or indented, with vesicular yet irregularly clumped chromatin and prominent macronucleoli (Fig. 2). Mitotic figures were frequent (Fig. 2, insert).

The histological sections of the omental biopsy showed sheets of malignant cells with scattered small aggregates of mature lymphocytes and focal tumor necrosis. The tumor cells (Fig. 3) were large, variable in size, polygonal to oval in shape with abundant, bright eosinophilic to glassy cytoplasm. Focal area of the tumor cells showed finely vacuolated cytoplasm. The nuclei were round to oval, vesicular with single prominent eosinophilic nucleoli. Nuclei were eccentrically situated in many cells. A brush-like border of cell membrane surface was appreciated in some cells at higher magnification. Binucleated or multinucleated cells were commonly seen. Mitotic figures were 1-2 per high power field. Using immunohistochemical stains on the biopsy, the malignant cells were diffusely positive for pan-cytokeratin, vimentin, and EMA, mildly positive with calretinin in $<25 \%$ of the tumor and negative for HMBE-1, CD45, HMB45, desmin, muscle 


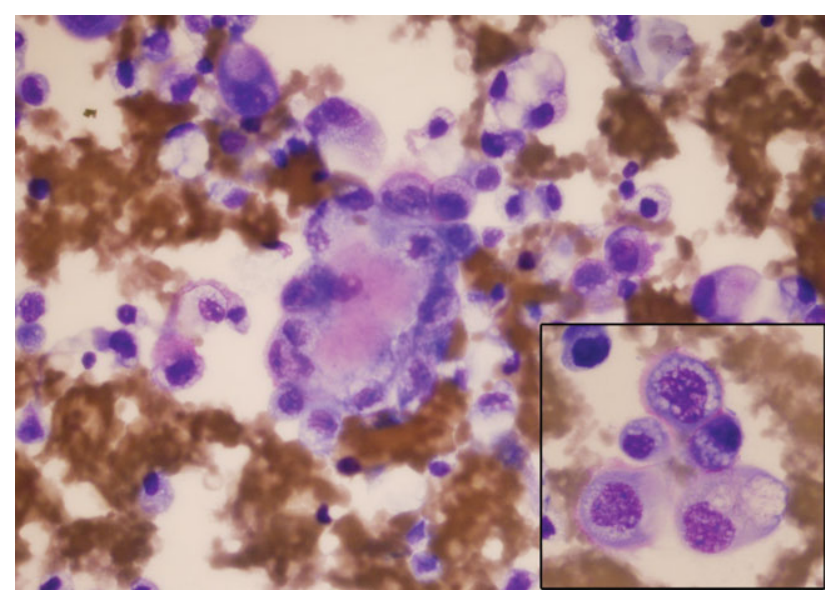

Fig. 4. Case 2: Cells are pleomorphic with abundant and highly vacuolated cytoplasm. A cell cluster forms a pseudoacinar structure surrounding globular eosinophilic extracellular material, and scalloped or hobnail border. Membranous brush border is noted (insert). Diff-Quik stain, $\times 600$. [Color figure can be viewed in the online issue, which is available at wileyonlinelibrary.com.]

specific actin, progesterone receptor, estrogen receptor, CD68, human placental lactogen and alpha fetoprotein.

Case 2. A 64-year-old white female school teacher initially presented with significant weight gain and abdominal distension on January 2006. The CT scan of her abdomen and pelvis performed in February revealed large amount of ascites and moderate amount of left pleural fluid. At outside hospital, the ascitic fluid was diagnosed as adenocarcinoma. Diagnostic laparoscopy was performed in March and revealed multiple peritoneal nodules which were sampled. The smears of peritoneal fluid and the slides of tissue biopsy were sent to us for consultation, and consequently diagnosed as deciduoid mesothelioma. The patient underwent several regimens of chemotherapy including six rounds of platinum and pemetrexed completed in July 2006. In February 2007, she developed ascites which was aspirated, submitted for cytological evaluation (current case) and diagnosed as recurrent mesothelioma. She consequently received nine cycles of gemcitabine completed in 2008, 37 cycles of doxorubicin liposomal completed January 2011 and 7 cycles of navelbine completed in August 2011. Her medical history is significant for hypertension, obesity, diabetes, buccal squamous cell carcinoma diagnosed in July 2009 and metastatic papillary thyroid carcinoma diagnosed in August 2010. She had history of asbestos exposure in her school and home. The patient is still alive in December 2011.

A total of $90 \mathrm{ml}$ of bloody ascitic fluid was obtained by peritoneocentesis for cytology. The smears were moderate in cellularity. The cells manifested as both single cells and small loosely cohesive clusters. Overall there was a wide variation in the size and shape of cells. Most cells were large, pleomorphic with abundant and highly

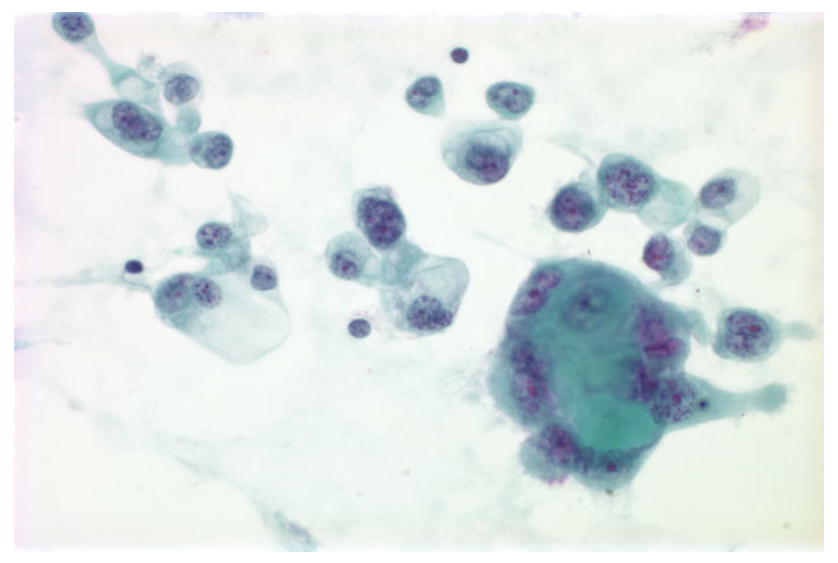

Fig. 5. Case 2: Cells exhibit pleomorphic nuclei, hyperchromatic, and coarse chromatin, with one to two prominent nucleoli. Groups of three to four cells often form multiple cell-to-cell windows. Disfiguring cytoplasmic vacuolization is often seen. Papanicolaou stain, $\times 600$. [Color figure can be viewed in the online issue, which is available at wileyonlinelibrary.com.]

vacuolated cytoplasm rendering a low $N / C$ ratio to most cells. Most cells showed fine cytoplasmic vacuoles. Some cells showed the classic dense cytoplasm but only rare cells had easily recognizable two tone staining (Fig. 4). At high magnification, many cells exhibited circumferential eosinophilic brush border (Fig. 4, insert). The nuclei were pleomorphic, hyperchromatic with coarse chromatin, irregular nuclear membrane, and occasionally prominent one to two nucleoli (Fig. 5). Nuclei were eccentrically situated in most cells. Double or multinucleated cells and mitosis were not uncommon. Some cells frequently presented as doublets and triplets with cell to cell articulation (windows). Two unusual features were noted in these articulated cells; first there was frequently striking difference in the size of the two opposing cells and second a large cell was sometimes articulated with two or more smaller cells at one pole (Figs. 5 and 6B). The clusters, usually composed of 5-12 cells each, were two dimensional, exhibited prominent scalloped or hob-nail borders. The cluster often formed a pseudoacinar pattern surrounding a globular extracellular material which stained pink on Diff-Quik and cyanophilic on Papanicolaou stain (Figs. 4 and 5). Histochemical stains for trichrome and modified Movat pentachrome were performed on the cell block to further characterize this globular material. The globules stained blue with trichrome and showed both yellow and pale green staining by Movat indicating both old and new collagen. ${ }^{11}$ Within the small cell clusters, the cytoplasmic vacuoles were prominent and frequently disfigured the groups (Fig. 5). Microcalcifications were also noted within some of these clusters (Fig. 6A). Because this fluid was collected after a course of chemotherapy, EMA and desmin were performed to rule out therapy related reactive changes. The atypical cells were strongly 


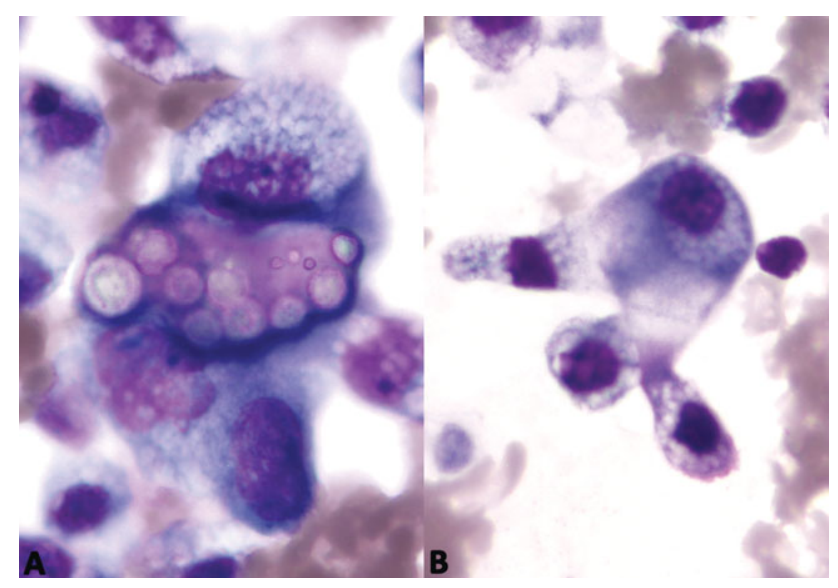

Fig. 6. Case 2: Microcalcifications are identified in some cell clusters (A). A large cell formed cell-to-cell windows with other three smaller cells (B). Diff-Quik stain, $\times 1,000$. [Color figure can be viewed in the online issue, which is available at wileyonlinelibrary.com.]

reactive to EMA and negative to desmin favoring its malignant nature. ${ }^{12}$ In addition the cellular features were compared to the previous biopsy and were found to be morphologically similar.

The omental biopsy showed predominantly sheets of tumor cells with focal necrosis. Focal areas showed dense fibrotic omentum infiltrated by tumor cells and lymphocytes. The tumor cells were large with abundant finely vacuolated cytoplasm, low $N / C$ ratio and indistinct cell borders (Fig. 7). Islands of tumor cells with dense eosinophilic cytoplasm were interspersed within the tissue sections. The nuclei were pleomorphic with hyperchromatic coarse chromatin, irregular nuclear membranes and inconspicuous nucleoli. Binucleated and multinucleated cells were common. Mitotic figures were not common. Multiple foci of psammoma bodies or calcifications were present in the histology sections. On immunohistochemical staining, the malignant cells were diffusely and strongly positive for AE1/3, CAM5.2; focally but strongly positive for CK5/6 and calretinin. The malignant cells were focally weakly positive for Ber-EP4 staining. The immunostains for melanoma markers HMB45, MART-1, Melan $\mathrm{A}$, and tyrosinase were all negative.

\section{Discussion}

Malignant mesothelioma (MM) is a relatively rare malignancy. The incidence of mesothelioma is reported to be 2,500 cases in the United States and 5,000 cases in Western Europe annually. Up to $80 \%$ of malignant pleural mesothelioma patients have history of asbestos exposure. ${ }^{13}$ Peritoneal MM accounts for $\sim 30 \%$ of MM. In the United States, its incidence is estimated to be 200-400 new cases annually. It can occur in any age group, although it mostly affects the 50- to 69-year age group. It is more common in men, possibly because of the higher

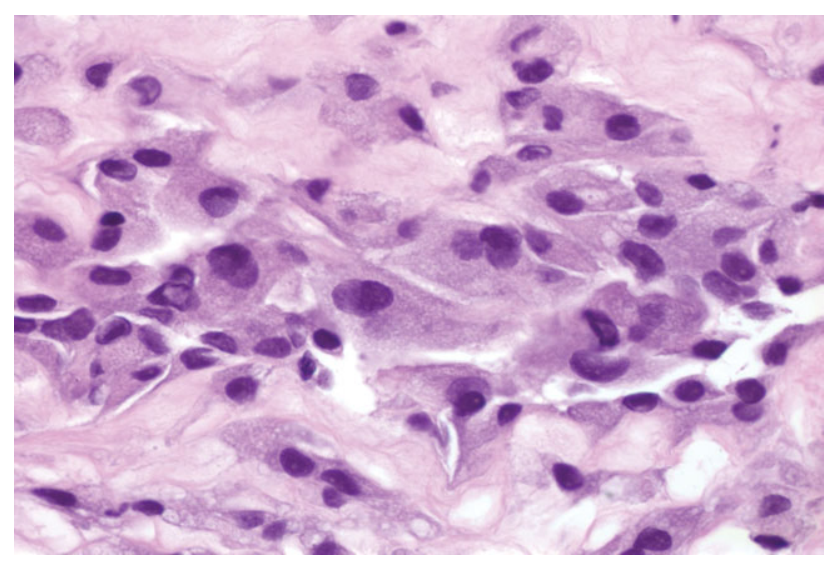

Fig. 7. Case 2: The cells are large, polygonal with voluminous, finely vacuolated cytoplasm, low $N / C$ ratio and indistinct cell borders. H\&E stain, $\times 400$. [Color figure can be viewed in the online issue, which is available at wileyonlinelibrary.com.]

male occupational exposure to asbestos. ${ }^{14}$ It is estimated that $57 \%$ of patients with a peritoneal MM have a history of asbestos exposure. ${ }^{15}$ However, other risk factors such as radiation exposure are also reported in association with mesothelioma. ${ }^{16,17}$ Histologically, there are three major variants of mesothelioma: epithelioid, sarcomatoid, and mixed (biphasic). Clinically, the presenting symptoms of mesothelioma tend to be insidious. Commonly these patients present with large serous effusions. ${ }^{18}$ Chemotherapy is usually the best option for patients with advanced disease. Cisplatin plus pemetrexed has demonstrated a significant survival advantage and is considered as firstline treatment for MM. ${ }^{19}$ Combination chemotherapy regimen of an antifolate (pemetrexed and raltitrexed) and a platinum-based (cisplatin) agent has shown a median survival of about $12-14$ months. ${ }^{19}$ Patients with malignant pleural mesothelioma have a 5-year survival of $15 \%{ }^{19}$ Because of the peritoneal confinement of MM and low occurrence of metastasis, an approach consisting of cytoreductive surgery and perioperative intraperitoneal chemotherapy has been introduced as a curative treatment option over the last decade with an overall 5-year survival rate of $29-63 \% .^{20}$

The MDM was first reported in a 13-year-old girl with primary peritoneal $\mathrm{MM}^{2}$ Contrary to the original belief that it is a malignancy that occurs mainly in young females, it is now known that MDM could occur in both sexes, at any age and with or without history of asbestos exposure. $^{1,3,4,6}$ Clinically, the pleura and peritoneum are most common sites for primary MDM. ${ }^{21-23}$ However, MDM of other locations such as pericardium and spermatic cord have also been reported. ${ }^{1,6}$ MDM tends to be aggressive with shorter survival. ${ }^{4,23}$ There are no large survival studies for MDM due to its rarity. However, the survival data collected from MDM case reports shows that approximately more than half the patients died within 
Table I. Comparison of Cytomorphological Features in MDM, MM, and ADC

\begin{tabular}{|c|c|c|c|}
\hline Feature & $M D M$ & $M M$ & $A D C$ \\
\hline Cellularity & Moderate & Very high & Usually high \\
\hline \multicolumn{4}{|l|}{ Smear pattern } \\
\hline Cell type & One cell type & One cell type & Two cell type \\
\hline Windows & Double or triple windows & Frequent & No \\
\hline Cell-in-cell & Occasionally & Frequent & Occasional \\
\hline Cellular clasping & No or rare & Prominent & No \\
\hline \multicolumn{4}{|l|}{ Individual cell } \\
\hline Size & Small to large & Small to gigantic & Enlarged, less variation \\
\hline \multirow[t]{3}{*}{ Cytoplasm } & Abundant & Abundant & Rare abundant \\
\hline & Most fine vacuoles & Rare & Variable \\
\hline & Rare two-tone & Two-tone & One-tone \\
\hline Submembranous vacuoles & Present & Present & Absent \\
\hline Brush border & Present & Present & Absent \\
\hline \multicolumn{4}{|l|}{ Nuclei } \\
\hline Size & Marled enlarged & Marked enlarge & Enlarged \\
\hline Chromatin & Vesicular and coarse & Variable & Vesicular or coarse \\
\hline Membrane & Marked irregularity & Variable irregularity & Marked irregularity \\
\hline Nucleoli & Eosinophilic macronucleoli & Macronucleoli & Can be prominent \\
\hline$N / C$ ratio & Low & Low & High \\
\hline Double or multinucleated cell & Frequent & Frequent & Rare \\
\hline Mitosis & Frequent & Inconspicuous & Variable \\
\hline \multicolumn{4}{|l|}{ Cell clusters } \\
\hline Size & Small & Large & Usually small \\
\hline Shape & Loose clusters, 2D & Spheres, morules, 3D & Tight clusters, spheres, 3D \\
\hline Border & Scalloped or hob-nail & Knobby or scalloped border & Smooth common border \\
\hline
\end{tabular}

MDM: malignant deciduoid mesothelioma; MM: malignant mesothelioma; ADC: adenocarcinoma.

1 year, and the remaining patients died within $1-3$ years with only rare patients surviving $>4$ years after the initial diagnosis. ${ }^{1}$ Our patients were different in gender and age and both presented as primary peritoneal MDM. The young male patient had no reported history of asbestos exposure although a family history of MM in both his uncle and grandmother suggest environmental exposure. He received no further therapy and was deceased 5 months after the diagnosis. The elderly female patient had history of asbestos exposure both at her home during childhood and at her work place. She received several rounds of extensive chemotherapy, and is still alive 69 months after the initial diagnosis suggesting that chemotherapy had a positive impact on her prognosis and survival. Overall, the clinical picture of these two patients is consistent with that reported in the literature for MDM. ${ }^{1,3,6}$

Although the cytological features in our two cases were different, they showed some common features. Both peritoneal fluids were moderately cellular with predominantly single cells and few loose cell clusters. Both showed striking variation in size and shape and an overall high degree of nuclear pleomorphism. While mesothelial features were not easily recognized, search at higher magnification revealed cells showing cellular articulation or window-like spaces, cell within cell, abundant cytoplasm with occasional cells exhibiting two tone staining, or endoectoplasmic differentiation, low $N / C$ ratio, fine submembranous glycogen vacuoles, and brush cytoplasmic borders. These features are consistent with those of MM. ${ }^{24}$ However, other features such as cellular clasping, morules and spheres were absent particularly in case 1 . The histological features in both cases were similar to those described in the literature. ${ }^{2-4,25}$ On the other hand, our two cases showed some morphological differences and triggered different differential diagnoses (Table I). While case 1 had predominantly single cells with dense cytoplasm, case 2 contained an admixture of single cells and small clusters with highly vacuolated cytoplasm and surrounding collagenous cores as confirmed by special stains, features that are distinctly different from those previously reported. ${ }^{9,10}$ Despite the high degree of pleomorphism in case 2, its mitotic activity was much lower than that in case 1 . The main differential diagnosis in case 1 especially considering the age and sex should include sarcoma, seminoma or melanoma. Morphologically, sarcoma in effusions may round up and lose their spindle shapes. They are usually vimentin positive and may react with other stains depending on their line of differentiation. Seminoma or germ cell tumors are another extremely rare malignancy in effusion, but tend to have fragile cytoplasm with fine vacuoles and lack the variation in size. Melanoma is also rare and tends to present with features similar to those seen on aspirates such as intranuclear pseudoinclusions, dusty cytoplasm and in occasional cases melanin. While adenocarcinoma (ADC) is always a possibility, it is far less likely in this age. Immunohistochemical staining should easily distinguish these entities.

The primary differential in case 2 was metastatic ADC due to the pleomorphic nuclear features, cellular clusters, and marked cytoplasmic vacuolation. Indeed the first fluid 
specimen before the tissue biopsy was misdiagnosed as ADC in initial workup. Subtle mesothelial features were noted in this case at high magnification and there was only one cell population. In contrast in ADC, an alien population is frequently seen in the background of reactive mesothelium. Carcinoma cells tend to show little variation in cellular size and shapes and frequently three dimensional clusters with smooth common border rather than scalloped borders (Table I). Because of the history of chemotherapy in this case and some of the therapy related changes depicted in the cells as multinucleation, cytomegally, and large cytoplasmic vacuoles, reactive mesothelium had to be ruled out. It should be noted however, that therapy changes are very seldom seen in effusions. In addition, the features seen in the second effusion was morphologically similar to those seen in the omental biopsy and previous fluid. The positive reaction of these cells to EMA favored their malignant nature. ${ }^{12}$

On the basis of our two cases, MDM tends to present with high degree of pleomorphism unlike the epithelioid MM which generally exhibit subtle atypia and can easily be underdiagnozed as reactive. Although some mesothelial features as described above can be detected and should alert the reviewer to the possibility of a mesothelioma variant, not all MM features are seen and therefore, a definitive diagnosis cannot be rendered without ancillary tests. Immunohistochemical panel is again essential to confirm the mesothelial origin of these cells. Calrectinin, D2-40 and cytokeratin 5/6 are commonly used as mesothelial markers. While the latter two markers also react with non-mesothelial tumors, they are useful when used in a panel that includes mesothelial markers and markers that rule out carcinoma. EMA and desmin play a role in the differential diagnosis between RM and MM. MM has strong membranous EMA staining in majority of cases which is rarely present in RM. Desmin is preferentially expressed in RM and is lost expression in MM. In addition, several new markers have been reported and may be useful in the differentiating of RM and MM. Isoform glucose transporter, GIUT-1, is normally undetectable by immunohistochemistry in normal epithelial tissues and benign tumors, and is increased in expression in a variety of malignancies. GLUT-1 is expressed in $100 \%$ of pleural MM, $100 \%$ of lung squamous cell carcinoma and $93.35 \%$ of lung adenocarcinoma, while is not expressed in RM cells. $^{26} \mathrm{X}$-linked inhibitor of apoptosis protein (XIAP) is also reported for its values in the differential diagnosis of RM and MM. Strong XIAP staining is often observed in MM, while RM is usually negative for XIAP by immunostaining. ${ }^{27,28}$ Other ancillary studies such as chromosome deletion analysis for $1 p, 3 p, 6 q, 9 p$, and $22 q$ by fluorescence in situ hybridization has shown high sensitivity and high specificity in distinguishing reactive mesothelial cells and mesothelioma. ${ }^{29,30}$ Ber-EP4 and Moc-31 are often included in the panel to differentiate ADC from RM or MM. However, these two markers can be focally positive in up to $20 \%$ of the MM. ${ }^{31,32}$ PAX8 is a very highly sensitive and specific marker for peritoneal Mullerian tumor and is essentially negative in $\mathrm{RM}$ or $\mathrm{MM}^{33}$

In conclusion, we described the cytomorphological and histological features in two MDM cases arising in the peritoneal cavity. The cytomorphology of MDM could be subtle and easily overlooked resulting in misdiagnosis. However, careful examination should reveal the subtle mesothelial features and trigger proper ancillary testing rendering an accurate diagnosis possible.

\section{References}

1. Ustun H, Astarci HM, Sungu N, Ozdemir A, Ekinci C. Primary malignant deciduoid peritoneal mesothelioma: A report of the cytohistological and immunohistochemical appearances. Diagn Cytopathol 2011;39:402-408.

2. Talerman A, Montero JR, Chilcote RR, Okagaki T. Diffuse malignant peritoneal mesothelioma in a 13-year-old girl. Report of a case and review of the literature. Am J Surg Pathol 1985;9:73-80.

3. Ordonez NG. Epithelial mesothelioma with deciduoid features: Report of four cases. Am J Surg Pathol 2000;24:816-823.

4. Shia J, Erlandson RA, Klimstra DS. Deciduoid mesothelioma: A report of 5 cases and literature review. Ultrastruct Pathol 2002;26: 355-363.

5. Henley JD, Loehrer PJ, Sr, Ulbright TM. Deciduoid mesothelioma of the pleura after radiation therapy for Hodgkin's disease presenting as a mediastinal mass. Am J Surg Pathol 2001;25:547-548.

6. Shanks JH, Harris M, Banerjee SS, et al. Mesotheliomas with deciduoid morphology: A morphologic spectrum and a variant not confined to young females. Am J Surg Pathol 2000;24: 285-294.

7. Nascimento AG, Keeney GL, Fletcher CD. 1994. Deciduoid peritoneal mesothelioma. An unusual phenotype affecting young females. Am J Surg Pathol 1994;18:439-445.

8. Serio G, Scattone A, Pennella A, et al. Malignant deciduoid mesothelioma of the pleura: Report of two cases with long survival. Histopathology 2002;40:348-352.

9. Gillespie FR, van der Walt JD, Derias N, Kenney A. Deciduoid peritoneal mesothelioma. A report of the cytological appearances. Cytopathology 2001;12:57-61.

10. Reis-Filho JS, Pope LZ, Milanezi F, Balderrama CM, Serapiao MJ, Schmitt FC. Primary epithelial malignant mesothelioma of the pericardium with deciduoid features: Cytohistologic and immunohistochemical study. Diagn Cytopathol 2002;26:117-122.

11. Russell HK, Jr. A modification of Movat's pentachrome stain. Arch Pathol 1972;94:187-191.

12. Hasteh F, Lin GY, Weidner N, Michael CW. The use of immunohistochemistry to distinguish reactive mesothelial cells from malignant mesothelioma in cytologic effusions. Cancer Cytopathol 2010;118:90-96.

13. Cugell DW, Kamp DW. Asbestos and the pleura: A review. Chest 2004; 125:1103-1117.

14. Bridda A, Padoan I, Mencarelli R, Frego M. 2007. Peritoneal mesothelioma: A review. MedGenMed 2007;9:32.

15. Antman KH, Pomfret EA, Aisner J, MacIntyre J, Osteen RT, Greenberger JS. Peritoneal mesothelioma: Natural history and response to chemotherapy. J Clin Oncol 1983;1:386-391.

16. Goodman JE, Nascarella MA, Valberg PA. Ionizing radiation: a risk factor for mesothelioma. Cancer Causes Control 2009;20:12371254. 
17. Metz-Flamant C, Guseva Canu I, Laurier D. Malignant pleural mesothelioma risk among nuclear workers: A review. J Radiol Prot 2011;31:9-23.

18. Ismail-Khan R, Robinson LA, Williams CC, Jr, Garrett CR, Bepler G, Simon GR. Malignant pleural mesothelioma: A comprehensive review. Cancer Control 2006;13:255-263.

19. Vogelzang NJ, Rusthoven JJ, Symanowski J, et al. Phase III study of pemetrexed in combination with cisplatin versus cisplatin alone in patients with malignant pleural mesothelioma. J Clin Oncol 2003;21:2636-2644.

20. Mirarabshahii P, Pillai K, Chua TC, Pourgholami MH, Morris DL. Diffuse malignant peritoneal mesothelioma-An update on treatment. Cancer Treat Rev 2011;38:605-612.

21. Santos C, Gamboa F, Fradinho F, Pego A, Carvalho L, Bernardo J. Deciduoid pleural mesothelioma-A rare entity in a young woman. Rev Port Pneumol 2012 Mar 15. [Epub ahead of print].

22. Okonkwo A, Musunuri S, Diaz L, Jr, Bedrossian C, Stryker S, Rao S. Deciduoid mesothelioma: A rare, distinct entity with unusual features. Ann Diagn Pathol 2001;5:168-171.

23. Mourra N, de Chaisemartin C, Goubin-Versini I, Parc R, Flejou JF. Malignant deciduoid mesothelioma: A diagnostic challenge. Arch Pathol Lab Med 2005;129:403-406.

24. Michael CW.Maligant mesothelioma. In: Davidson B, Pinar F, Michael CW, editors. Serous effusions. London: Springer; 2012. p 79-98.

25. Talerman A. Deciduoid or pseudodecidual mesothelioma. Am J Surg Pathol 2000;24:1179.
26. Kato Y, Tsuta K, Seki K, et al. Immunohistochemical detection of GLUT-1 can discriminate between reactive mesothelium and malignant mesothelioma. Mod Pathol 2007;20:215-220.

27. Wu M, Sun Y, Li G, et al. Immunohistochemical detection of XIAP in mesothelium and mesothelial lesions. Am J Clin Pathol 2007; 128:783-787.

28. Kleinberg L, Lie AK, Florenes VA, Nesland JM, Davidson B. Expression of inhibitor-of-apoptosis protein family members in malignant mesothelioma. Hum Pathol 2007;38:986-994.

29. Granados R, Cibas ES, Fletcher JA. Cytogenetic analysis of effusions from malignant mesothelioma. A diagnostic adjunct to cytology. Acta Cytol 1994;38:711-717.

30. Xiao S, Renshaw A, Cibas ES, Hudson TJ, Fletcher JA. Novel fluorescence in situ hybridization approaches in solid tumors. Characterization of frozen specimens, touch preparations, and cytological preparations. Am J Pathol 1995;147:896-904.

31. Pu RT, Pang Y, Michael CW. Utility of WT-1, p63, MOC31, mesothelin, and cytokeratin (K903 and CK5/6) immunostains in differentiating adenocarcinoma, squamous cell carcinoma, and malignant mesothelioma in effusions. Diagn Cytopathol 2008;36:20-25.

32. Lozano MD, Panizo A, Toledo GR, Sola JJ, Pardo-Mindan J. Immunocytochemistry in the differential diagnosis of serous effusions: A comparative evaluation of eight monoclonal antibodies in Papanicolaou stained smears. Cancer 2001;93:68-72.

33. Wiseman W, Michael CW, Roh MH. Diagnostic utility of PAX8 and PAX2 immunohistochemistry in the identification of metastatic Mullerian carcinoma in effusions. Diagn Cytopathol 2011;39:651-656. 